\title{
Synthesis and Evaluation of Urea and Thiourea Derivatives of Oxazolidinones as Antibacterial Agents
}

\author{
Sunil Kumar Reddy AaramadaKa, ${ }^{*, a}$ Mrinal kanthi Guha, ${ }^{a}$ Ganesh Prabhu, ${ }^{a}$ Suvarna Ganesh. Kini, ${ }^{b}$ \\ and Magesh VIJAYAN ${ }^{a}$ \\ ${ }^{a}$ New Drug Discovery, Orchid Chemicals \& Pharmaceuticals Ltd.; Plot No 476/14, Old Mahabalipuram Road, \\ Sholinganallur, Chennai-600019, Tamilnadu, India: and ${ }^{b}$ Department of Pharmaceutical Chemistry, Manipal College of \\ Pharmaceutical Sciences; Manipal-576104, Karnataka, India. Received May 20, 2006; accepted October 30, 2006
}

Urea and thiourea derivatives of oxazolidinones were synthesized and their inhibitory activity (MIC) was determined on the bacterial strains which includes clinical isolates and quality control organisms. The structure activity relationships were studied and a 3D-QSAR model was built using Genetic Function Approximation. Interestingly found that electron withdrawing groups at the ortho position of the phenyl ring enhances the activity.

Key words oxazolidinone; antimicrobial agent; piperazine; structure activity relationship

Since 1970 antibiotic resistance has been a major concern especially in regard to multi drug resistant gram-positive bacteria. Examples of such organisms include methicillin resistant Staphylococcus aureus (MRSA), methicillin resistant Staphylococcus epidermidis (MRSE), vancomycin resistant Enterococcus (VRE), quinolone resistant Staphylococcus aureus (QRSA) and penicillin resistant Streptococcus pneumoniae (PRSP). ${ }^{1-4)}$ These organisms are responsible for many life threatening infections like pneumonia, surgical infections, soft tissue infections, abscesses, heart valve infections and infections of the blood. ${ }^{5)}$

In 1978, DuPont demonstrated a series of oxazolidinones having in vitro antibacterial activity against human pathogens. The result of continuous investigations on this series resulted in DUP 105 \& DUP 721 (Fig. 1) potent in vitro and in vivo antibacterial agents. ${ }^{6-9)}$

In 1987, DUP 721 was selected as an antibacterial new candidate for clinical trials. However, its further development was discontinued due to toxicity observed in phase-I clinical trials. ${ }^{10)}$ The special features of their antibacterial activity attracted much attention and prompted studies on oxazolidinones in a number of laboratories. These are a novel class of synthetic antibacterial agents with activity against anaerobic and gram-positive bacteria. ${ }^{11)}$ They represent the first new antimicrobial class to be released onto to market over a decade and are structurally unrelated to any other compounds currently in the market.

The Pharmacia-Upjohn scientists were able to identify
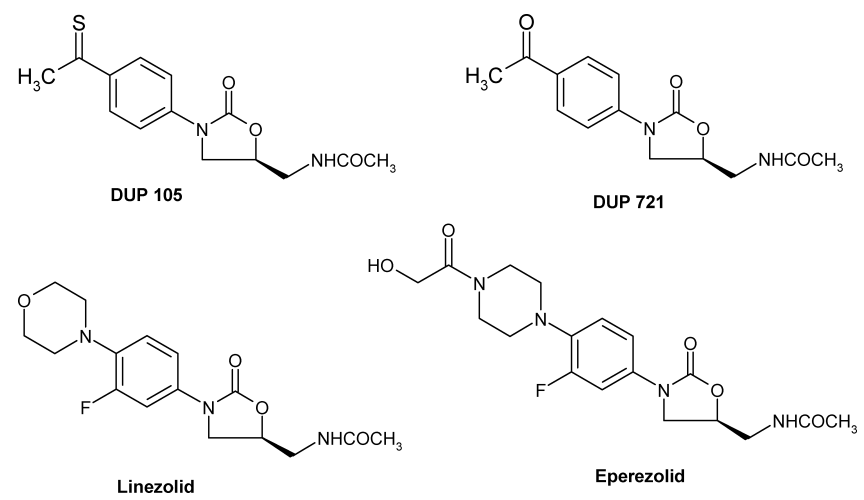

Fig. 1 two drug candidates for human studies linezolid and eperezolid $^{10)}$ (Fig. 1).

The linezolid which was discovered is well known as the first promising candidate of oxazolidinones and works effectively against numerous serious gram-positive pathogens like methicillin resistant Staphylococcus aureus (MRSA), Staphylococcus epidermidis, vancomycin resistant enterococci (VRE) and penicillin resistant pneumococci. ${ }^{1,11)}$

Oxazolidinones are an appealing class of antimicrobials due to their unique bacteriostatic mechanism of action. [They have been shown selectively and uniquely to bind 50s ribosomal subunit and inhibit translation], lack of cross resistance with other antibacterial agents, good oral bioavailability, potential for structural manipulation. ${ }^{1,5,12)}$ New agents with greater potency and new spectra of activity could arise from the further modification of oxazolidinone nucleus. The potential of this oxazolidinones stimulated an exploratory chemical program in our Drug Discovery Laboratories.

Chemistry The synthesis of oxazolidinone basic moiety (compound A) was accomplished by the procedures, which have been described previously ${ }^{8,10)}$ (Chart 1).

The thioanisole $(0.264 \mathrm{~g}, 2.12 \mathrm{mmol})$ was added to a solution of compound $\mathbf{A}(0.250 \mathrm{~g}, 0.51 \mathrm{mmol})$ in dichloromethane $(15 \mathrm{ml})$ under stirring at $0{ }^{\circ} \mathrm{C}$ and continued the stirring for $6 \mathrm{~h}$. To the resulted reaction mass trifluoroacetic acid $(0.96 \mathrm{~g}, 8.4 \mathrm{mmol})$ in DCM $(10 \mathrm{ml})$ was added drop wise under stirring at the same condition. The completion of the reaction was confirmed by TLC, using ethyl acetate-hexane as solvent system. Then the reaction mass was neutralized with triethylamine $(2.17 \mathrm{~g}, 21.4 \mathrm{mmol})$ at $0{ }^{\circ} \mathrm{C}$ to yield the compound $\mathbf{B}$.

Example: The triethylamine $(0.283 \mathrm{~g}, 2.77 \mathrm{mmol})$ followed by 4-chlorophenyl isothiocyanate $(0.107 \mathrm{~g}, 0.63 \mathrm{mmol})$ was added to the solution of compound $\mathbf{B}$ (obtained above) in DCM $(15 \mathrm{ml})$ at $0-4{ }^{\circ} \mathrm{C}$ under stirring. Continued the stirring at the same temperature for $15 \mathrm{~min}$ and further stirring continued at $30^{\circ} \mathrm{C}$ for $2 \mathrm{~h}$. After completion of the reaction, the resulted reaction mass was sonicated for $15 \mathrm{~min}$ after addition of water $(20 \mathrm{ml})$ and hexane $(20 \mathrm{ml})$ to yield white color final compound (compound 1, $244 \mathrm{mg}, 86 \%$ ) (Chart 2).

Further following the procedure mentioned above the compounds $\mathbf{2}-\mathbf{1 3}$ were synthesized using compound $\mathbf{B}$ (Table 1). 


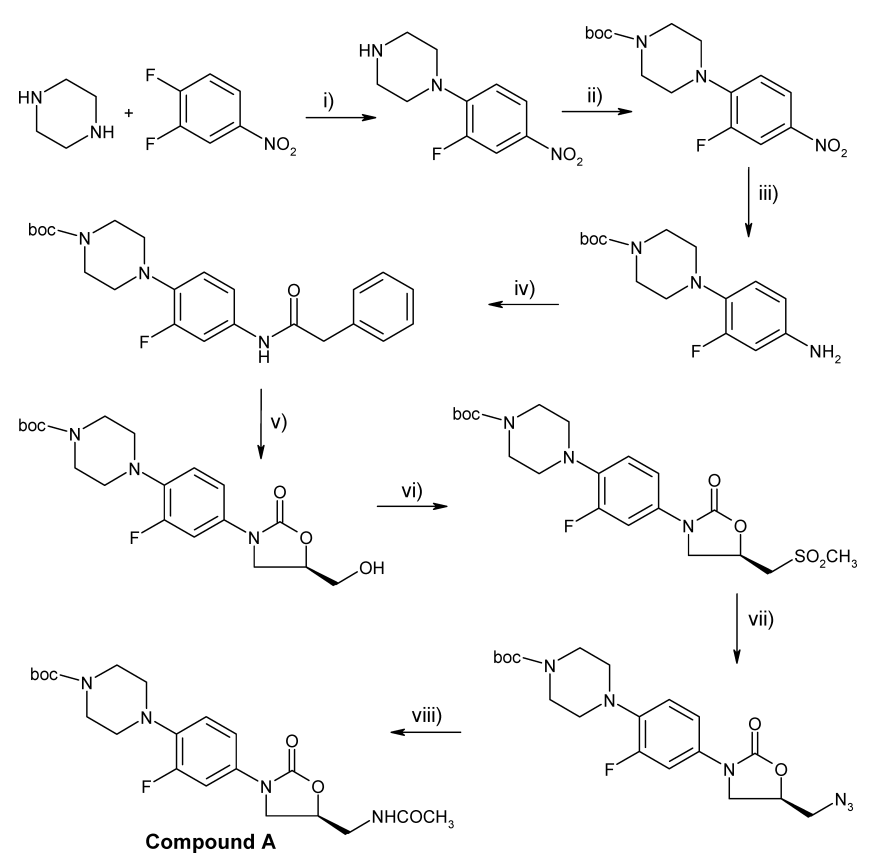

Reagents: i) $\mathrm{CH}_{3} \mathrm{CN}, 70^{\circ} \mathrm{C}$; ii) $\mathrm{BOC}$ anhydride, $\mathrm{THF}, 0-25^{\circ} \mathrm{C}$; iii) $10 \% \mathrm{Pd} / \mathrm{C}$, EtOAc, 40 psi; iv) $\mathrm{Cbz}$, DMA, THF, $0-25^{\circ} \mathrm{C}$; v) $n$-butyl lithium, $R$ - $(-)$-glycidyl butyrate, THF, $-78^{\circ} \mathrm{C}$; vi) $\mathrm{CH}_{3} \mathrm{SO}_{2} \mathrm{Cl}, \mathrm{NEt}_{3}, \mathrm{DCM}, 0-25^{\circ} \mathrm{C}$; vii) $\mathrm{NaN}_{3}, \mathrm{DMF}, 60-$ $70{ }^{\circ} \mathrm{C}$; viii) $10 \% \mathrm{Pd} / \mathrm{C}$, EtOAc, pyridine, acetic anhydride.

Chart 1
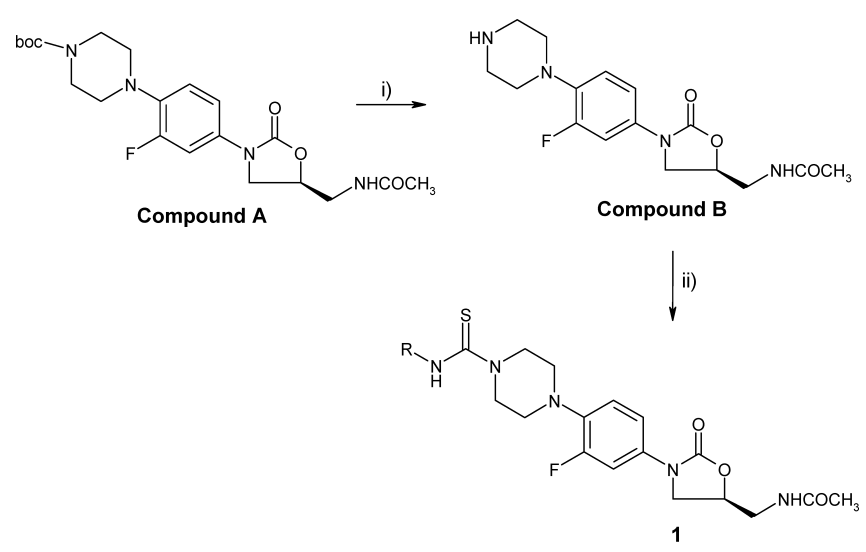

Reagents: i) TFA, thioanisole, $\mathrm{DCM}, \mathrm{NEt}_{3}$; ii) $\mathrm{NEt}_{3}$, 4-chlorophenyl isothiocyanate, DCM.

Chart 2

Structure Activity Relationship The antibacterial oxazolidinone pharmacophore (Fig. 2) consists of an oxazolidinone ring, ('A' ring), 'B' ring (aromatic ring) attached at nitrogen of the 'A' ring, ' $\mathrm{C}$ ' ring (piperazinyl ring) is present as the substituent on ' $\mathrm{B}$ ' ring. Oxazolidinone pharmacophore also tolerates another substituted aromatic ring (' $D$ ' ring) attached to a piperazinyl ring (' $\mathrm{C}$ ' ring) as a substituent. The substitution at 3rd and 5th position and stereochemistry of the substitution at 5th position of the oxazolidinone ring plays a critical role in the antimicrobial activity. The 5-(S)configuration is necessary for activity. The substituent amidomethyl group at C-5 imparts improved activity. And 3$N$-aryl group (Ring 'B') is essential for activity. ${ }^{12)}$ In view of this, Ring A, Ring B and Ring $C$ moieties were kept unchanged.

The synthesized molecules (Table 1) were tested for in
Table 1. Structures of Synthesized Compounds
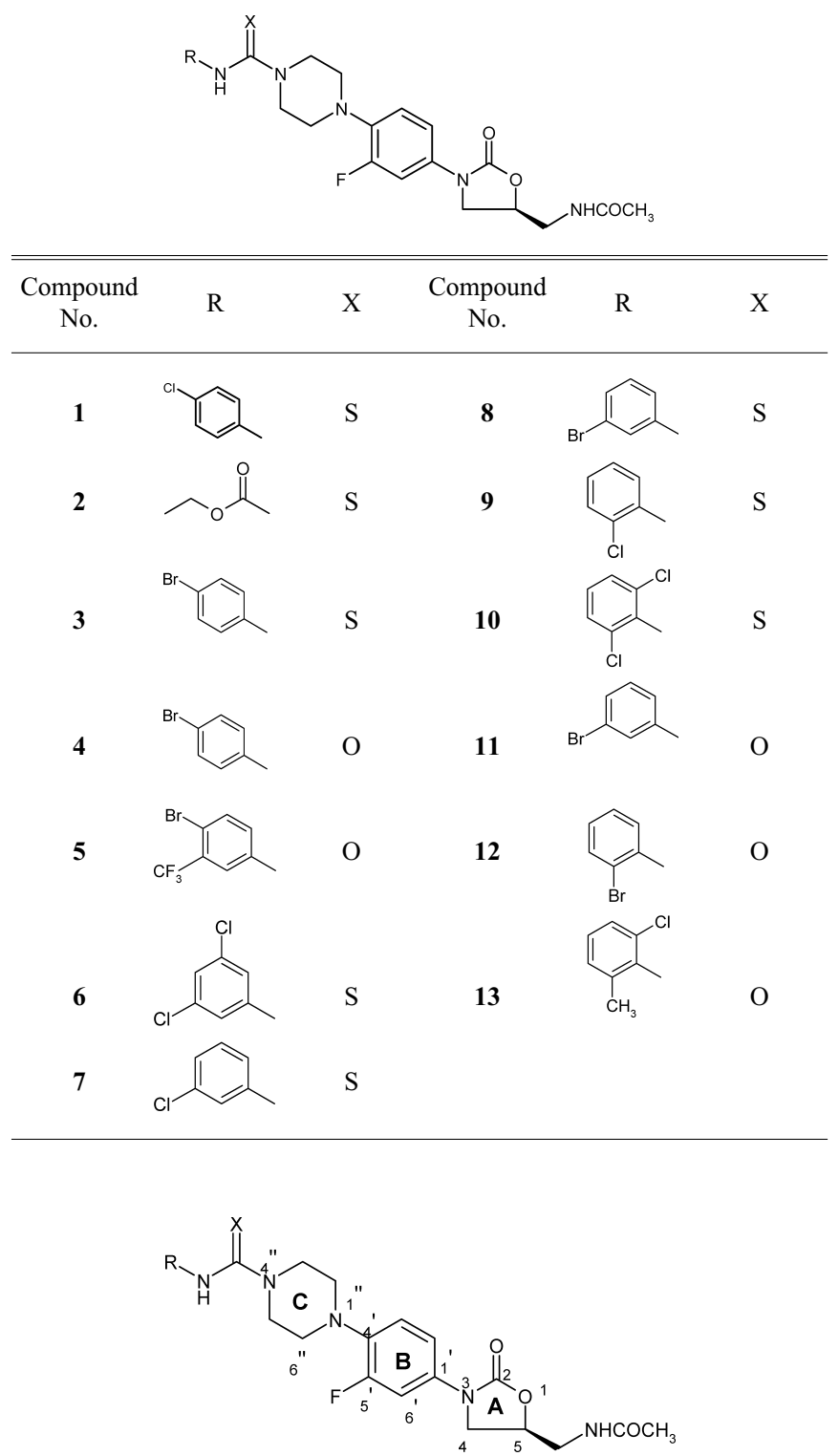

Fig. 2

vitro antibacterial activity against clinical isolates and quality control organisms using linezolid as reference compound. The minimum inhibitory concentrations (MICs) of these compounds were listed in Table 2. For convenience these compounds were discussed as urea derivatives and thiourea derivatives.

Interestingly it is found that the ortho and meta substituted phenyl urea derivatives $(\mathbf{1 1}, \mathbf{1 2})$ showed comparable activity against gram-positive organisms and moderate activity against gram-negative organisms to that of linezolid. As increasing the substitution at phenyl ring the potency seems to be decreased $(5, \mathbf{1 3})$. It was clear from the study that the mono halo substitution on ring D showed comparatively potent activity than the others. But $o$-chloro (1), di-o-chloro (6), $m$-chloro (7), $m$-bromo (8), $p$-chloro (9) and $p$-dichloro (10) substitution of $\mathrm{D}$ ring showed moderate activity against gram-positive organisms and exhibited poor activity against gram-negative organisms.

Thirteen urea and thiourea derivatives were synthesized 
Table 2. In Vitro Antibacterial Activity: Minimum Inhibitory Concentration $(\mu \mathrm{g} / \mathrm{ml})$

\begin{tabular}{|c|c|c|c|c|c|c|c|c|c|c|c|c|c|c|c|}
\hline S. No. & Organism & Suscep key & $\begin{array}{l}\text { Line- } \\
\text { zolid }\end{array}$ & 1 & 2 & 3 & 4 & 6 & 7 & 8 & 9 & 10 & 11 & 12 & 13 \\
\hline 1. & S. aureus MRO00013 & OSSA & 4 & 8 & 8 & 8 & 8 & 8 & 4 & 4 & 8 & 8 & 8 & 8 & 16 \\
\hline 2. & S. aureus MRO00055 & OSSA & 4 & 8 & 8 & 8 & 8 & 8 & 4 & 4 & 8 & 8 & 8 & 8 & 16 \\
\hline 3. & S. epidermidis MRO02046 & OSSE & 2 & 8 & 8 & 4 & 4 & 8 & 4 & 4 & 4 & 4 & 2 & 2 & 8 \\
\hline 4. & S. haemolyticus MRO02053 & OSCONS & 2 & 8 & 8 & 4 & 4 & 8 & 4 & 4 & 4 & 4 & 4 & 2 & 4 \\
\hline 5. & S. aureus MRO00001 & ORSA & 2 & 8 & 8 & 4 & 4 & 8 & 4 & 4 & 4 & 4 & 4 & 2 & 4 \\
\hline 6. & S. aureus MRO00003 & ORSA & 2 & 8 & 8 & 4 & 4 & 8 & 4 & 4 & 4 & 4 & 4 & 4 & 8 \\
\hline 7. & S. aureus MRO00030 & ORSA & 2 & 8 & 8 & 4 & 4 & 8 & 4 & 4 & 4 & 4 & 4 & 2 & 8 \\
\hline 8. & S. aureus MRO00048 & ORSA & 2 & 8 & 8 & 4 & 4 & 8 & 4 & 4 & 4 & 4 & 4 & 2 & 8 \\
\hline 9. & S. aureus MRO00059 & ORSA & 2 & 8 & 8 & 4 & 4 & 8 & 4 & 4 & 4 & 4 & 4 & 2 & 8 \\
\hline 10. & S. epidermidis MRO02002 & ORSE & 2 & 8 & 8 & 4 & 4 & 8 & 4 & 4 & 4 & 4 & 4 & 4 & 8 \\
\hline 11. & S. epidermidis MRO02045 & ORSE & 1 & 8 & 8 & 4 & 4 & 8 & 4 & 4 & 4 & 4 & 2 & 2 & 4 \\
\hline 12. & S. epidermidis MRO02095 & ORSE & 2 & 8 & 8 & 4 & 4 & 8 & 4 & 4 & 4 & 4 & 2 & 2 & 4 \\
\hline 13. & S. saprophyticus MRO02003 & ORCONS & 2 & 8 & 8 & 4 & 4 & 8 & 4 & 4 & 8 & 8 & 4 & 4 & 8 \\
\hline 14. & S. haemolyticus MRO02064 & ORCONS & 1 & 8 & 8 & 4 & 4 & 8 & 4 & 4 & 4 & 4 & 4 & 2 & 8 \\
\hline 15. & E. faecalis MRO04045 & VSE & 2 & 8 & 8 & 4 & 4 & 8 & 4 & 4 & 4 & 4 & 2 & 2 & 8 \\
\hline 16. & E. faecalis MRO04034 & VRE & 4 & 8 & 8 & 4 & 4 & 8 & 4 & 4 & 4 & 4 & 2 & 4 & 8 \\
\hline 17. & E. faecalis MRO04035 & VRE & 2 & 8 & 8 & 4 & 4 & 8 & 4 & 4 & 4 & 4 & 2 & 4 & 8 \\
\hline 18. & E. faecium MRO04036 & VRE & 4 & 8 & 8 & 4 & 4 & 8 & 4 & 4 & 4 & 4 & 2 & 4 & 8 \\
\hline 19. & E. faecium MRO04037 & VRE & 2 & 8 & 8 & 4 & 4 & 8 & 4 & 4 & 4 & 4 & 2 & 2 & 4 \\
\hline 20. & E. faecium MRO04038 & VRE & 2 & 8 & 8 & 4 & 4 & 8 & 4 & 4 & 4 & 4 & 2 & 2 & 8 \\
\hline 21. & E. faecalis ATCC51299 & Quality control & 2 & 8 & 4 & 4 & 8 & 4 & 4 & 4 & 4 & 2 & 2 & 4 & 8 \\
\hline 22. & E. faecium АTCC700221 & VRE & 2 & 8 & 8 & 4 & 4 & 8 & 4 & 4 & 4 & 4 & 2 & 2 & 4 \\
\hline 23. & E. faecalis ATCC29212 & Quality control & 2 & 8 & 8 & 4 & 4 & 8 & 4 & 4 & 4 & 4 & 2 & 2 & 4 \\
\hline 24. & S. aureus ATCC29213 & Quality control & 4 & 8 & 8 & 4 & 8 & 8 & 4 & 4 & 8 & 8 & 4 & 4 & 8 \\
\hline 25. & S. aureus ATCC43300 & Quality control & 4 & 8 & 8 & 4 & 8 & 8 & 4 & 4 & 4 & 4 & 4 & 4 & 8 \\
\hline
\end{tabular}

and the MIC was determined for all the derivatives except for compound 5. From the MIC values depicted in Table 2 it can be concluded that urea derivatives (compounds $4,11,12,13$ ) are more potent than thio urea derivatives (compounds $\mathbf{1}, \mathbf{2}$, $3,6,7,8,9,10)$.

3D QSAR To gain further insight into the structure-activity relationship of these oxazolidinone antibacterial and to derive a predictive three-dimensional quantitative structure activity relationship (3D-QSAR) model, we made use of the Genetic Function Approximation algorithm (GFA) in cerius 3 .

GFA algorithm offers a new approach to building structure activity models. It automates the search for QSAR models by combining a genetic algorithm with statistical modeling tools (Thousands of candidate models are created and tested during evolution only the superior model survives.). GFA has been successfully applied for generation of 3D-QSAR models which can be used for designing newer compounds and activity prediction prior to synthesis. ${ }^{3)}$ GFA predicted values are given in Table 3.

Study Set: MIC data against various strains analyzed for the standard linezolid and 11 molecules synthesized were presented (Table 2). MIC values for S. aureus MRO 00001 presents a range of value than others.

3D structures of molecules were constructed on a 3D computer graphics interface. Structures were improved by minimizing the conformational energy by molecular mechanics and semi empirical quantum mechanics. All the molecules were superimposed and they are aligned in a grid box. Interactive energies were calculated using $\mathrm{CH}_{3}$ as steric parameter and $\mathrm{H}^{+}$as electrostatic parameter their values are entered as descriptors.

Thousands of descriptors are generated among them only with $10 \%$ variance were used in generating the QSAR equa-
Table 3. GFA Predicted Activity

\begin{tabular}{cccr}
\hline \hline Compound & Activity & $\begin{array}{c}\text { GFA predicted } \\
\text { activity }\end{array}$ & $\begin{array}{c}\text { GFA residuals } \\
\text { activity }\end{array}$ \\
\hline $\mathbf{1}$ & -0.903 & -0.69230 & -0.210700 \\
$\mathbf{2}$ & -0.903 & -0.96803 & 0.065032 \\
$\mathbf{3}$ & -0.602 & -0.69605 & 0.094053 \\
$\mathbf{4}$ & -0.602 & -0.54992 & -0.052080 \\
$\mathbf{6}$ & -0.903 & -0.86088 & -0.042120 \\
$\mathbf{7}$ & -0.602 & -0.63015 & 0.028151 \\
$\mathbf{8}$ & -0.602 & -0.65741 & 0.055411 \\
$\mathbf{9}$ & -0.602 & -0.57939 & -0.022610 \\
$\mathbf{1 0}$ & -0.602 & -0.65248 & 0.050477 \\
$\mathbf{1 1}$ & -0.301 & -0.33976 & 0.038761 \\
$\mathbf{1 2}$ & -0.602 & -0.58945 & -0.012550 \\
Linezolid & -0.301 & -0.30906 & 0.008055 \\
\hline
\end{tabular}

tion.

GFA - correlates activity with descriptors and checks the stability of the QSAR model. Final sets of GFA models were analyzed statistically to select the best model.

The QSAR model obtained is given by the equation:

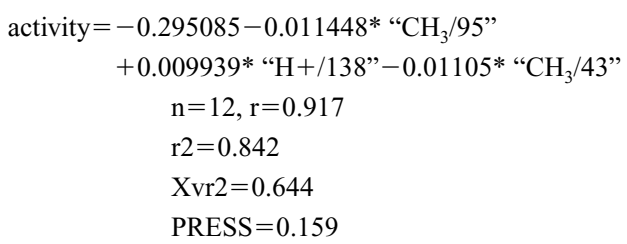

$\mathrm{n}$ is the number of compounds used in the study, $\mathrm{r}$ represents the correlation coefficient which is the measure of quality of fit of the model. $\mathrm{r} 2$ is the square of correlation coefficients. $\mathrm{Xvr} 2$ is cross-validated $\mathrm{r} 2$. PRESS predicted residual errors of sum of squares, which is the sum of squared deviations 
between predicted and activity molecules for every molecule. From this model it can be deduced that

- A hydrophobic or steric substitution at meta-position of the terminal phenyl ring towards the field point $\mathrm{CH}_{3} / 43$ is not favorable for activity.

- A hydrophobic or steric substitution at meta-position of the terminal phenyl ring towards the field point $\mathrm{CH}_{3} / 95$ may decrease the activity.

- Electropositive substitution is being favored at field point $\mathrm{H}+/ 138$, near the ortho-position of the terminal phenyl ring.

\section{Conclusion}

From the MIC data it can be concluded that the compounds $\mathbf{1 1}$ and $\mathbf{1 2}$ are having close activity to the comparator linezolid, whereas rest of the compounds are possessing moderate activity. The 3D-QSAR studies reveal that electropositive substitution at field point $\mathrm{H}+/ 138$ near the orthoposition of the terminal phenyl ring will increase the activity. Hence promising antibacterial agents can be synthesized by placing electron with drawing groups at the ortho-position of the phenyl ring.

\section{Experimental}

Melting points were determined on Buchi B-540 automatic melting point apparatus and are uncorrected. Infrared spectra were recorded in $\mathrm{KBr}$ on Perkin-Elmer Paragon-1000 model. ${ }^{1} \mathrm{H}-\mathrm{NMR}$ spectra were obtained in solvent DMSO- $d_{6}$ using Brukes-400 spectrometer, TMS as internal standard.

(S)- $N$-[3-\{ $N$-(4-Chlorophenyl)-4-(3-fluorophenyl)piperazine]-1-carbothioamido\}-2-oxooxazolidin-5-yl)methyl|acetamide (1) The triethylamine $(0.283 \mathrm{~g}, 2.77 \mathrm{mmol})$ followed by 4-chlorophenyl isothiocyanate $(0.107 \mathrm{~g}, 0.63 \mathrm{mmol})$ were added to the solution of compound $\mathbf{B}$ (obtained according to the procedure described in the chemistry section) in DCM $(15 \mathrm{ml})$ at $0-4{ }^{\circ} \mathrm{C}$ under stirring. Continued the stirring for $15 \mathrm{~min}$ at the same condition and further stirring continued at $30^{\circ} \mathrm{C}$ for $2 \mathrm{~h}$. After completion of the reaction, the resulted reaction mass was sonicated for $15 \mathrm{~min}$ after addition of water $(20 \mathrm{ml})$ and hexane $(20 \mathrm{ml})$ to yield white color final compound, (compound 1, $244 \mathrm{mg}, 86 \%),\left(\mathrm{mp} 210-214^{\circ} \mathrm{C}\right) \mathrm{IR}\left(\mathrm{cm}^{-1}\right): 3389$, 3301, 1724, 1652. MS: $(\mathrm{M}+1)^{+} 506,(\mathrm{M}+2)^{+} 507 .{ }^{1} \mathrm{H}-\mathrm{NMR}$ (DMSO- $\left.d_{6}\right): \delta$ $1.80(3 \mathrm{H}, \mathrm{s}), 3.08(4 \mathrm{H}, \mathrm{t}), 3.30(2 \mathrm{H}, \mathrm{t}), 3.68(1 \mathrm{H}, \mathrm{t}), 4.06(5 \mathrm{H}, \mathrm{q}), 4.7(1 \mathrm{H}$ m) $7.09(1 \mathrm{H}, \mathrm{t}), 7.18(1 \mathrm{H}, \mathrm{d}), 7.49(1 \mathrm{H}, \mathrm{d}), \mathrm{NH} 8.2(1 \mathrm{H}, \mathrm{bs}), 9.48(1 \mathrm{H}, \mathrm{s})$. Anal. Calcd for $\mathrm{C}_{23} \mathrm{H}_{25} \mathrm{ClFN}_{5} \mathrm{O}_{3} \mathrm{~S}$ : C, 57.08; H, 5.23; N, 12.10. Found: C, $57.03 ; \mathrm{H}, 5.1 ; \mathrm{N}, 12.00$.

(S)- $N$-[3-\{ $N$-(Ethoxycarbonyl)-4-(3-fluorophenyl)piperazine]-1-carbothioamido\}-2-oxooxazolidin-5-yl)methyl]acetamide (2) The triethylamine $(0.283 \mathrm{~g}, 2.77 \mathrm{mmol})$ followed by ethoxycarbonyl isothiocyanate $(0.082 \mathrm{~g}, 0.62 \mathrm{mmol})$ were added to the solution of compound $\mathbf{B}$ and further following the procedure described for compound $\mathbf{1}$ yielded the title compound (compound 2) as light yellow solid (162 mg, 62\%), (mp 134$\left.137^{\circ} \mathrm{C}\right)$. IR $\left(\mathrm{cm}^{-1}\right): 3280,3212,1728,1621$. MS: $(\mathrm{M}+1)^{+}$468.2. ${ }^{1} \mathrm{H}-\mathrm{NMR}$ (DMSO- $\left.d_{6}\right): \delta 1.82(3 \mathrm{H}, \mathrm{s}), 3.05(4 \mathrm{H}, \mathrm{t}), 3.32(3 \mathrm{H}, \mathrm{t}), 3.38(2 \mathrm{H}, \mathrm{t}), 3.7(2 \mathrm{H}$ $\mathrm{m}), 4.07(4 \mathrm{H}, \mathrm{t}), 4.2(1 \mathrm{H}, \mathrm{bs}), 4.7(1 \mathrm{H}, \mathrm{m}), 7.07(1 \mathrm{H}, \mathrm{t}), 7.17(1 \mathrm{H}, \mathrm{d}), 7.48$ $(1 \mathrm{H}, \mathrm{d}), \mathrm{NH}-8.32(1 \mathrm{H}, \mathrm{bs}), 10.18(1 \mathrm{H}, \mathrm{s})$. Anal. Calcd for $\mathrm{C}_{20} \mathrm{H}_{26} \mathrm{FN}_{5} \mathrm{O}: \mathrm{C}$, 53.76; H, 5.94; N, 13.20. Found: C, 53.60; H, 6.00; N, 13.00 .

(S)- $N$-[3-\{ $N$-(4-Bromophenyl)-4-(3-fluorophenyl)piperazine]-1-carbothioamido\}-2-oxooxazolidin-5-yl)methyl|acetamide (3) The triethylamine $(0.283 \mathrm{~g}, 2.77 \mathrm{mmol})$ followed by para-bromophenyl isothiocyanate $(0.130 \mathrm{~g}, 0.60 \mathrm{mmol})$ were added to the solution of compound $\mathbf{B}$ and further following the procedure described for compound $\mathbf{1}$ afforded the title compound (compound 3) $(162 \mathrm{mg}, 51 \%)$ an off white solid, (mp 226-229 ${ }^{\circ} \mathrm{C}$ ). IR $\left(\mathrm{cm}^{-1}\right): 3389,3303,1728$. MS: $(\mathrm{M}+1)^{+} 551 .{ }^{1} \mathrm{H}-\mathrm{NMR}\left(\mathrm{DMSO}-d_{6}\right): \delta$ $1.82(3 \mathrm{H}, \mathrm{s}), 3.05(4 \mathrm{H}, \mathrm{t}), 3.39(2 \mathrm{H}, \mathrm{t}), 3.68(1 \mathrm{H}, \mathrm{t}), 4.06(5 \mathrm{H}, \mathrm{q}), 4.7(1 \mathrm{H}$, m) $7.09(1 \mathrm{H}, \mathrm{t}), 7.18(1 \mathrm{H}, \mathrm{d}), 7.27(2 \mathrm{H}, \mathrm{d}), 7.47(2 \mathrm{H}, \mathrm{d}), \mathrm{NH}-8.25(1 \mathrm{H}, \mathrm{bs})$, $9.48(1 \mathrm{H}, \mathrm{S})$. Anal. Calcd for $\mathrm{C}_{23} \mathrm{H}_{25} \mathrm{BrFN}_{5} \mathrm{O}_{3} \mathrm{~S}: \mathrm{C}, 52.08 ; \mathrm{H}, 4.77 ; \mathrm{N}, 11.04$. Found: C, 52.00; H, 4.5; N,11.0.

(S)- $N$-[3-\{ $N$-(4-Bromophenyl)-4-(3-fluorophenyl)piperazine]-1-carboxyamido\}-2-oxooxazolidin-5-yl)methyl|acetamide (4) The triethylamine $(0.283 \mathrm{~g}, 2.77 \mathrm{mmol})$ followed by para-bromophenyl isocyanate $(0.113 \mathrm{~g}, 0.57 \mathrm{mmol})$ were added to the solution of compound $\mathbf{B}$ and further following the procedure described for compound $\mathbf{1}$ afforded the title compound (compound 4) $(250 \mathrm{mg}, 81 \%)$ a white solid, $\left(\mathrm{mp} 214-216^{\circ} \mathrm{C}\right)$. IR $\left(\mathrm{cm}^{-1}\right): 3359,3275,1716,1672 . \mathrm{MS}:(\mathrm{M}+1)^{+} 536,\left(\mathrm{M}+\mathrm{NH}_{4}\right)^{+} 553 .{ }^{1} \mathrm{H}-$ NMR (DMSO- $\left.d_{6}\right): \delta 1.81(3 \mathrm{H}, \mathrm{s}), 2.96(4 \mathrm{H}, \mathrm{t}), 3.05(2 \mathrm{H}, \mathrm{m}), 3.35(3 \mathrm{H}$, m), $3.57(4 \mathrm{H}, \mathrm{t}), 3.68(1 \mathrm{H}, \mathrm{t}), 4.0(1 \mathrm{H}, \mathrm{t}), 4.7(1 \mathrm{H}, \mathrm{m}), 7.08(1 \mathrm{H}, \mathrm{t}), 7.15$ $(1 \mathrm{H}, \mathrm{d}), 7.34(5 \mathrm{H}, \mathrm{m}), \mathrm{NH}-8.2(1 \mathrm{H}, \mathrm{bs}), 8.73(1 \mathrm{H}, \mathrm{s})$. Anal. Calcd for $\mathrm{C}_{23} \mathrm{H}_{25} \mathrm{BrFN}_{5} \mathrm{O}_{4}: \mathrm{C}, 53.78 ; \mathrm{H}, 4.92 ; \mathrm{N}, 11.40$. Found: $\mathrm{C}, 53.70 ; \mathrm{H}, 5.0 ; \mathrm{N}$, 11.30 .

(S)- $N$-[3-\{ $N$-(4-Bromo-3-trifluoromethylphenyl)-4-(3-fluorophenyl)piperazine]-1-carboxyamido\}-2-oxooxazolidin-5-yl)methyl]acetamide (5) The triethylamine $(0.283 \mathrm{~g}, 2.77 \mathrm{mmol})$ followed by 4-chloro-3-trifluoromethylphenyl isocyanate $(0.126 \mathrm{~g}, 0.56 \mathrm{mmol})$ were added to the solution of compound $\mathbf{B}$ and further following the procedure described for compound $\mathbf{1}$ afforded the title compound (compound 5) (223 mg, 70\%) a white solid, (mp 246-248 $\left.{ }^{\circ} \mathrm{C}\right)$. IR $\left(\mathrm{cm}^{-1}\right): 3405,3272,1729,1661$. MS: $(\mathrm{M}+1)^{+} 558$, $(\mathrm{M}+2)^{+} 559 .{ }^{1} \mathrm{H}-\mathrm{NMR}\left(\mathrm{DMSO}-d_{6}\right): \delta 1.81(3 \mathrm{H}, \mathrm{s}), 2.98(4 \mathrm{H}, \mathrm{t}), 3.37(2 \mathrm{H}$, t), $3.61(4 \mathrm{H}, \mathrm{t}), 3.66(1 \mathrm{H}, \mathrm{t}), 4.04(1 \mathrm{H}, \mathrm{t}), 4.68(1 \mathrm{H}, \mathrm{m}), 7.06(1 \mathrm{H}, \mathrm{t}), 7.15$ $(1 \mathrm{H}, \mathrm{d}), 7.46(1 \mathrm{H}, \mathrm{d}), 7.55(1 \mathrm{H}, \mathrm{d}), 7.77(1 \mathrm{H}, \mathrm{d}), 8.04(1 \mathrm{H}, \mathrm{s}), \mathrm{NH}-8.22(1 \mathrm{H}$, bs), $9.04(1 \mathrm{H}, \mathrm{s})$. Anal. Calcd for $\mathrm{C}_{24} \mathrm{H}_{27} \mathrm{ClF}_{4} \mathrm{~N}_{5} \mathrm{O}_{4}: \mathrm{C}, 52.03 ; \mathrm{H}, 4.37 ; \mathrm{N}$, 10.55. Found: C, $52.00 ; \mathrm{H}, 4.40 ; \mathrm{N}, 10.45$.

(S)- $N$-[3-\{N-(3,5-Dichlorophenyl)-4-(3-fluorophenyl)piperazine]-1carbothioamido\}-2-oxooxazolidin-5-yl)methyl]acetamide (6) The triethylamine $(0.283 \mathrm{~g}, 2.77 \mathrm{mmol})$ followed by 3,5-dichlorophenyl isothiocyanate $(0.116 \mathrm{~g}, 0.56 \mathrm{mmol})$ were added to the solution of compound $\mathbf{B}$ and further following the procedure described for compound $\mathbf{1}$ afforded the title compound (compound 6) $(223 \mathrm{mg}, 70 \%)$ a white solid, $\left(\mathrm{mp} 220-223^{\circ} \mathrm{C}\right)$. IR $\left(\mathrm{cm}^{-1}\right): 3357,3278,1726,1713$. MS: $(\mathrm{M}+1)^{+} 540,(\mathrm{M}+2)^{+} 542 .{ }^{1} \mathrm{H}-$ NMR (DMSO- $\left.d_{6}\right): \delta 1.83(3 \mathrm{H}, \mathrm{s}), 3.06(4 \mathrm{H}, \mathrm{t}), 3.39(2 \mathrm{H}, \mathrm{t}), 3.7(1 \mathrm{H}, \mathrm{t})$, $4.06(5 \mathrm{H}, \mathrm{q}), 4.7(1 \mathrm{H}, \mathrm{m}), 7.11(1 \mathrm{H}, \mathrm{t}), 7.18(1 \mathrm{H}, \mathrm{d}), 7.3(1 \mathrm{H}, \mathrm{d}), 7.45$ $(2 \mathrm{H}, \mathrm{d}), 7.46(1 \mathrm{H}, \mathrm{d}), \mathrm{NH}-8.25(1 \mathrm{H}, \mathrm{t}), 9.6(1 \mathrm{H}, \mathrm{s})$. Anal. Calcd for $\mathrm{C}_{23} \mathrm{H}_{24} \mathrm{Cl}_{2} \mathrm{FN}_{5} \mathrm{O}_{3} \mathrm{~S}: \mathrm{C}, 53.12 ; \mathrm{H}, 4.66 ; \mathrm{N}, 11.26$. Found: C, 53.00; H, 4.43; N, 11.2 .

(S)- $N$-[3-\{N-(3-Chlorophenyl)-4-(3-fluorophenyl)piperazine]-1-carbothioamido\}-2-oxooxazolidin-5-yl)methyl]acetamide (7) The triethylamine $(0.283 \mathrm{~g}, 2.77 \mathrm{mmol})$ followed by 3-chlorophenyl isothiocyanate $(0.097 \mathrm{~g}, 0.57 \mathrm{mmol})$ were added to the solution of compound $\mathbf{B}$ and further following the procedure described for compound $\mathbf{1}$ afforded the title compound (compound 7) $(118 \mathrm{mg}, 40 \%)$ an off white solid, $\left(\mathrm{mp} 206-208^{\circ} \mathrm{C}\right)$. IR $\left(\mathrm{cm}^{-1}\right): 3339,3281,1729,1650$. MS: $(\mathrm{M}+1)^{+} 506\left(\mathrm{M}+\mathrm{NH}_{4}\right)^{+} 523 .{ }^{1} \mathrm{H}-$ NMR (DMSO- $\left.d_{6}\right): \delta 1.83(3 \mathrm{H}, \mathrm{s}), 3.06(4 \mathrm{H}, \mathrm{t}), 3.39(2 \mathrm{H}, \mathrm{t}), 3.68(1 \mathrm{H}, \mathrm{t})$, $4.06(5 \mathrm{H}, \mathrm{q}), 4.7(1 \mathrm{H}, \mathrm{m}), 7.09(3 \mathrm{H}, \mathrm{m}), 7.28(2 \mathrm{H}, \mathrm{m}), 7.44(1 \mathrm{H}, \mathrm{s}), 7.49$ $(1 \mathrm{H}, \mathrm{d}), \mathrm{NH}-8.23(1 \mathrm{H}, \mathrm{bs}), 9.5(1 \mathrm{H}, \mathrm{s})$. Anal. Calcd for $\mathrm{C}_{23} \mathrm{H}_{25} \mathrm{ClFN}_{5} \mathrm{O}_{3} \mathrm{~S}: \mathrm{C}$, $57.08 ; \mathrm{H}, 5.23$; N, 12.10. Found: C, 57.00; H, 5.29; N, 12.32 .

(S)- $N$-[3-\{N-(3-Bromophenyl)-4-(3-fluorophenyl)piperazine]-1-carbothioamido\}-2-oxooxazolidin-5-yl)methyl]acetamide (8) The triethylamine $(0.283 \mathrm{~g}, 2.77 \mathrm{mmol})$ followed by 3-bromophenyl isothiocyanate $(0.116 \mathrm{~g}, 0.54 \mathrm{mmol})$ were added to the solution of compound $\mathbf{B}$ and further following the procedure described for compound $\mathbf{1}$ afforded the title compound (compound 8) $(152 \mathrm{mg}, 48 \%)$ light yellow solid, (mp 190-192 ${ }^{\circ} \mathrm{C}$ ). IR $\left(\mathrm{cm}^{-1}\right): 3281,3095,1730,1583$. MS: $(\mathrm{M}+1)^{+} 550,(\mathrm{M}+2)^{+} 552 .{ }^{1} \mathrm{H}_{-}$ NMR (DMSO- $\left.d_{6}\right): \delta 1.83(3 \mathrm{H}, \mathrm{s}), 3.06(4 \mathrm{H}, \mathrm{t}), 3.49(2 \mathrm{H}, \mathrm{t}), 3.68(1 \mathrm{H}, \mathrm{t})$, $4.06(5 \mathrm{H}, \mathrm{q}), 4.7(1 \mathrm{H}, \mathrm{m}), 4.11(5 \mathrm{H}, \mathrm{m}), 7.49(2 \mathrm{H}, \mathrm{t}), \mathrm{NH}-8.24(1 \mathrm{H}, \mathrm{t}), 9.5$ $\left(1 \mathrm{H}\right.$, s). Anal. Calcd for $\mathrm{C}_{23} \mathrm{H}_{25} \mathrm{BrFN}_{5} \mathrm{O}_{3} \mathrm{~S}$ : C, 52.08; H, 4.77; N, 11.04 . Found: C, 52.00; H, 4.0; N, 11.0 .

(S)- $N$-[3-\{N-(2-Chlorophenyl)-4-(3-fluorophenyl)piperazine]-1-carbothioamido\}-2-oxooxazolidin-5-yl)methyl]acetamide (9) The triethylamine $(0.283 \mathrm{~g}, 2.77 \mathrm{mmol})$ followed by 2-chlorophenyl isothiocyanate $(0.097 \mathrm{~g}, 0.57 \mathrm{mmol})$ were added to the solution of compound $\mathbf{B}$ and further following the procedure described for compound $\mathbf{1}$ afforded the title compound (compound 9) $(225 \mathrm{mg}, 78 \%)$ an off white solid, (mp 138-140 ${ }^{\circ} \mathrm{C}$ ). IR $\left(\mathrm{cm}^{-1}\right)$ : 3390, 3290, 1748, 1657. MS: $(\mathrm{M}+1)^{+} 506,(\mathrm{M}+2)^{+} 507 .{ }^{1} \mathrm{H}-$ NMR (DMSO- $\left.d_{6}\right): \delta 1.83(3 \mathrm{H}, \mathrm{S}), 3.06(5 \mathrm{H}, \mathrm{q}), 3.39(2 \mathrm{H}, \mathrm{t}), 3.69(1 \mathrm{H}, \mathrm{t})$ $4.09(4 \mathrm{H}, \mathrm{t}), 4.69(1 \mathrm{H}, \mathrm{m}), 7.13(2 \mathrm{H}, \mathrm{m}), 7.27(3 \mathrm{H}, \mathrm{m}), 7.48(2 \mathrm{H}, \mathrm{t}), \mathrm{NH}$ $8.24(1 \mathrm{H}, \mathrm{bs}), 9.35(1 \mathrm{H}, \mathrm{s})$. Anal. Calcd for $\mathrm{C}_{23} \mathrm{H}_{25} \mathrm{ClFN}_{5} \mathrm{O}_{3} \mathrm{~S}: \mathrm{C}, 57.08 ; \mathrm{H}$ $5.23 ; \mathrm{N}, 12.10$. Found: $\mathrm{C}, 57.00 ; \mathrm{H}, 5.39 ; \mathrm{N}, 12.30$.

(S)- $N$-[3-\{N-(2,6-Dichlorophenyl)-4-(3-fluorophenyl)piperazine]-1carbothioamido\}-2-oxooxazolidin-5-yl)methyl|acetamide (10) The triethylamine $(0.283 \mathrm{~g}, 2.77 \mathrm{mmol})$ followed by 2,6-dichlorophenyl isothiocyanate $(0.116 \mathrm{~g}, 0.56 \mathrm{mmol})$ were added to the solution of compound $\mathbf{B}$ and further following the procedure described for compound $\mathbf{1}$ afforded the title compound (compound 10) $(160 \mathrm{mg}, 51 \%)$ a white solid, $\left(\mathrm{mp} 215-218^{\circ} \mathrm{C}\right)$. IR $\left(\mathrm{cm}^{-1}\right): 3329,3282,1755,1655$. MS: $(\mathrm{M}+1)^{+} 540(\mathrm{M}+2)^{+} 542 .{ }^{1} \mathrm{H}-$ NMR (DMSO- $\left.d_{6}\right): \delta 1.83(3 \mathrm{H}, \mathrm{s}), 3.06(4 \mathrm{H}, \mathrm{t}), 3.39(2 \mathrm{H}, \mathrm{t}), 3.69(1 \mathrm{H}, \mathrm{t})$, $4.07(5 \mathrm{H}, \mathrm{q}), 4.7(1 \mathrm{H}, \mathrm{m}), 7.1(2 \mathrm{H}, \mathrm{m}), 7.3(1 \mathrm{H}, \mathrm{t}), 7.49(3 \mathrm{H}, \mathrm{m}), \mathrm{NH}-8.2$ 
(1H, bs), $9.4(1 \mathrm{H}, \mathrm{s})$. Anal. Calcd for $\mathrm{C}_{23} \mathrm{H}_{24} \mathrm{Cl}_{2} \mathrm{FN}_{5} \mathrm{O}_{3} \mathrm{~S}$ : C, 53.12; H, 4.66; $\mathrm{N}, 11.26$. Found: C, 53.00; H, 4.49; N, 11. 33 .

$(S)-N$-[3-\{ $N$-(3-Bromophenyl)-4-(3-fluorophenyl)piperazine]-1-carboxyamido\}-2-oxooxazolidin-5-yl)methyl]acetamide (11) The triethylamine $(0.283 \mathrm{~g}, 2.77 \mathrm{mmol})$ followed by 3-bromophenyl isothiocyanate $(0.113 \mathrm{~g}, 0.57 \mathrm{mmol})$ were added to the solution of compound $\mathbf{B}$ and further following the procedure described for compound $\mathbf{1}$ afforded the title compound (compound 11) $(157 \mathrm{mg}, 59 \%)$ a white solid, (mp 184-186 $\left.{ }^{\circ} \mathrm{C}\right)$. IR $\left(\mathrm{cm}^{-1}\right): 3430,3282,1730,1630$. MS: $(\mathrm{M}+1)^{+} 534(\mathrm{M}+2)^{+} 536 .{ }^{1} \mathrm{H}-\mathrm{NMR}$ $\left(\right.$ DMSO- $\left.d_{6}\right): \delta 1.83(3 \mathrm{H}, \mathrm{s}), 2.99(4 \mathrm{H}, \mathrm{t}), 3.38(2 \mathrm{H}, \mathrm{t}), 3.61(4 \mathrm{H}, \mathrm{t}), 3.68$ $(1 \mathrm{H}, \mathrm{t}), 4.0(1 \mathrm{H}, \mathrm{t}), 4.7(1 \mathrm{H}, \mathrm{m}), 7.08(2 \mathrm{H}, \mathrm{t}), 7.18(2 \mathrm{H}, \mathrm{t}), 7.44(1 \mathrm{H}, \mathrm{t})$ $7.52(1 \mathrm{H}, \mathrm{d}), 7.8(1 \mathrm{H}, \mathrm{s}), \mathrm{NH}-8.2(1 \mathrm{H}, \mathrm{bs}), 8.78(1 \mathrm{H}, \mathrm{s})$. Anal. Calcd for $\mathrm{C}_{23} \mathrm{H}_{25} \mathrm{BrFN}_{5} \mathrm{O}_{4}: \mathrm{C}, 53.78 ; \mathrm{H}, 4.92 ; \mathrm{N}, 11.40$. Found: $\mathrm{C}, 53.60 ; \mathrm{H}, 5.0 ; \mathrm{N}$, 11.30 .

$(S)-N$-[3-\{ $N$-(2-Bromophenyl)-4-(3-fluorophenyl)piperazine]-1-carboxyamido\}-2-oxooxazolidin-5-yl)methyl]acetamide (12) The triethylamine $(0.283 \mathrm{~g}, 2.77 \mathrm{mmol})$ followed by 3-bromophenyl isothiocyanate $(0.113 \mathrm{~g}, 0.60 \mathrm{mmol})$ was added to the solution of compound $\mathbf{B}$ and further following the procedure described for compound $\mathbf{1}$ afforded the title compound (compound 12) $(157 \mathrm{mg}, 51 \%)$ a white solid, (mp 223-226 $\left.{ }^{\circ} \mathrm{C}\right)$. IR $\left(\mathrm{cm}^{-1}\right): 3427,3284,1741,1665$. MS: $\left.(\mathrm{M}+1)^{+} 534(\mathrm{M}+2)^{+}\right)$536. ${ }^{1} \mathrm{H}-\mathrm{NMR}$ (DMSO- $\left.d_{6}\right): \delta 1.83(3 \mathrm{H}, \mathrm{s}), 3.0(4 \mathrm{H}, \mathrm{t}), 3.39(2 \mathrm{H}, \mathrm{t}), 3.61(4 \mathrm{H}, \mathrm{t}), 3.68(1 \mathrm{H}$, t), $4.06(1 \mathrm{H}, \mathrm{t}), 4.7(1 \mathrm{H}, \mathrm{m}), 7.07(2 \mathrm{H}, \mathrm{m}), 7.17(1 \mathrm{H}, \mathrm{d}), 7.32(1 \mathrm{H}, \mathrm{t}), 7.47$ $(2 \mathrm{H}, \mathrm{t}), 7.63(1 \mathrm{H}, \mathrm{d}), 8.2(2 \mathrm{H}, \mathrm{m})$. Anal. Calcd for $\mathrm{C}_{23} \mathrm{H}_{25} \mathrm{BrFN}_{5} \mathrm{O}_{4}: \mathrm{C}, 52.08$; $\mathrm{H}, 4.77 ; \mathrm{N}, 11.04$. Found: $\mathrm{C}, 52.00 ; \mathrm{H}, 4.60 ; \mathrm{N}, 11.0$.

(S)- $N$-[3-\{ $N$-(2-Chloro-6-methylphenyl)-4-(3-fluorophenyl)piperazine]-1-carboxyamido\}-2-oxooxazolidin-5-yl)methyl]acetamide (13) The triethylamine $(0.283 \mathrm{~g}, 2.77 \mathrm{mmol})$ followed by 2-chloro-6-methylphenyl isothiocyanate $(0.078 \mathrm{~g}, 0.42 \mathrm{mmol})$ were added to the solution of compound $\mathbf{B}$ and further following the procedure described for compound $\mathbf{1}$ afforded the title compound (compound 13) $(128 \mathrm{mg}, 44 \%)$ a white solid, (mp 207-209 $\left.{ }^{\circ} \mathrm{C}\right)$. IR $\left(\mathrm{cm}^{-1}\right)$ : 3346, 3092, 1731, 1630. MS: $(\mathrm{M}+1)^{+}$, 504.3, $\left(\mathrm{M}+\mathrm{NH}_{4}\right)^{+}, 521.27 .{ }^{1} \mathrm{H}-\mathrm{NMR}\left(\mathrm{DMSO}-d_{6}\right): \delta 1.83(3 \mathrm{H}, \mathrm{s}), 2.2(3 \mathrm{H}$, s), $2.99(4 \mathrm{H}, \mathrm{t}), 3.39(2 \mathrm{H}, \mathrm{m}), 3.6(4 \mathrm{H}, \mathrm{t}), 3.68(1 \mathrm{H}, \mathrm{t}), 4.06(1 \mathrm{H}, \mathrm{t}), 4.7(1 \mathrm{H}$, m), $7.12(4 \mathrm{H}, \mathrm{m}), 7.31(1 \mathrm{H}, \mathrm{d}), 7.49(1 \mathrm{H}, \mathrm{d}), \mathrm{NH}-8.2(2 \mathrm{H}, \mathrm{s})$. Anal. Calcd for $\mathrm{C}_{24} \mathrm{H}_{27} \mathrm{ClFN}_{5} \mathrm{O}_{4}: \mathrm{C}, 57.91 ; \mathrm{H}, 5.49 ; \mathrm{N}, 11.75$. Found: $\mathrm{C}, 58.00 ; \mathrm{H}, 5.3 ; \mathrm{N}$, 11.8 .

Acknowledgements One of the authors A. Sunil Kumar Reddy deeply acknowledges Dr. G. Om Reddy, Dr. U. P. Senthil Kumar and Dr. Uma Ra- machandran for their helpful discussions. He is grateful to Dr. S. K. Agarwal and Dr. Y. Kamalakar Rao for their valuable suggestions. The authors wish to thank the Department of Analytical Research and Development and Intellectual Patent Management, O. C. P. L. We extend our thanks to Dr. K. Narasimhan, S. S. Solanki and G. Satish Kumar for their timely assistance.

\section{References and Notes}

1) Sullivan P., Candidate P. D., SPAHS Drug Information Service, The University of Mountana, 3, 8 (1999).

2) Kumar S. N., Srinivas D., Khera S. M., Mamidi S. V., Rateem A. M., Das J., Iqbal J., Rajagopalan R., J. Med. Chem., 45, 3953-3962 (2002).

3) Karki R. G., Kulkarni V. M., Bioorg. Med. Chem., 9, 3153-3160 (2001).

4) Gregory W. A., Brittelli D. R., Wang J. L. C., Wuonola A. M., McRipley J. R., Eustice C. D., Eberly S. V., Bartholomew T. P., Slee M. A., Forbes M., J. Med. Chem., 32, 1673-1681 (1989).

5) Livermore D. M, International Symposium on Antimicrobial Agents and Resistance, Apr. 13, 2001, pp. 192-200.

6) Gregory W. A., Brittelli D. R., Wang J. L., Wuonola A. M., McRipley J. R., Eustice C. D., Eberly S. V., Bartholomew T. P., Slee M. A., Forbes M., J. Med. Chem., 33, 2569-2578 (1990).

7) Barbachyn M. R., Hutchinson D. K., Brickner J. S., Cyanamon H. M., Kilburn O. J., Klemans P. S., Glickman E. S., Grega C. K., Hendges K. S., Toops S. D., Ford W. C., Zorenko E. G., J. Med. Chem., 39, 680685 (1996).

8) Tucker J. A., Allwine D. A., Grega C. K., Barbachyn R. M., Klock L. J., Adamski L. J., Brickner J. S., Hutchinson K. D., Ford W. C., Zurenko E. G., Conradi A. R., Burton S. P., Jensen M. R., J. Med. Chem., 41, 3727-3735 (1998).

9) Tokoyama R., Takahashi Y., Tomita Y., Tsubouchi M., Iwasaki N., Kado N., Okezak E., Nagata O., Chem. Pharm. Bull., 49, 361-367 (2001).

10) Kim C. S., Chang S. J., Korean J. Med. Chem., 7, 145-153 (1997).

11) Diekema D. J., Jones R. N., Lancet, 1975-1982 (2001).

12) Gadwood R. C., Shinabarger D. A., Ann. Reports Med. Chem., 35, $135-144$ (2000).

13) Agarwal S. K., Guha M. K., Pandey S., Satyanarayanan K., Samuel M. M., U. S. Patent 0070526 A 1, (2005). 